\title{
Uso de Twitter en docencia: estudio bibliométrico
}

\section{Juan V. Oltra Gutiérrez ${ }^{a}$, Fernando J. Garrigos-Simón ${ }^{b}$, Yeamduan Narangajavana ${ }^{c}$, y José Onofre Montesa Andrés ${ }^{d}$ \\ a b , ${ }^{\mathrm{d}}$ UniversitatPolitècnica de València, jvoltra@omp.upv.es, ${ }^{\mathrm{c} U n i v e r s i t a t ~ J a u m e ~ I ~}$}

\begin{abstract}
Resumen
En la presente comunicación se expone el resumen de un estudio bibliométrico realizado sobre el uso de Twitter en la docencia, empleando para ello la base de datos de utilizando la base de datos de la Web of Science, identificando los artículos más relevantes clasificada por artículos, autores, y países.
\end{abstract}

Palabras clave:Bibliometría; Twitter; Docencia.

\begin{abstract}
In thepresentcommunicationthesummary of a bibliometricstudymadeonthe use of Twitter in teachingisexposed, usingforthisthedatabase of usingtheWeb of Sciencedatabase, identifyingthemostrelevantarticlesclassifiedbyarticles, authors, and countries.
\end{abstract}

Keywords:Bibliometrics; Twitter; Teaching.

\section{Introducción}

Siendo el uso docente de las redes sociales en general, y de Twitter en particular uno de los campos de trabajo que ocupan a los investigadores (Garrigos-Simón, Oltra Gutiérrez, \& Narangajavana, 2016) su repercusión real es algo de lo que, a menos que nos sumerjamos en la literatura científica especializada, no resulta posible cuantificar.

El presente trabajo pretende, mediante medición de fuentes, empleando la base de datos de la Web Of Sience (en adelante WOS) (Clarivate Analytics), dar una visión cuantitativa del uso de Twitter en la docencia, identificando los artículos más relevantes clasificada por artículos, autores, y países.

En el presente trabajo describiremos, en el apartado "Objetivos" los elementos que van a ser estudiados, delimitando el espacio temporal donde focalizaremos el estudio. En el 
apartado "Estudio" someramente los pasos seguidos y en "Resultados", el apartado más prolijo, daremos cuenta de los datos obtenidos, de los que se ofrece una visión simplificada en "Conclusiones".

\section{Objetivos}

Saber cuántas veces se ha citado un artículo es un indicador principal al medir los resultados de una investigación (Luo, Sun, Erdt, Raamkumar, \& Theng, 2018), o la calidad científica de instituciones (Cetto, A., 2012); pero no solo sirven este tipo de métricas para cuantificar la bondad de una investigació o de grupos de investigadores, sino que, como dicen (Galindo Rodríguez \& Arbinaga, 2018), atendiendo al recorrido histórico reflejado en las revistas especializadas, puede afirmarse que éstas aportan una gran cantidad de datos a la hora de analizar cuál ha sido el desarrollo científico de cualquier disciplina.

La bibliometría a menudo es cuestionada por su facilidad de ser manipulada, como nos recuerdan (Alhuay Quispe \& Pacheco Mendoza, 2018), (Chinchilla-Rodríguez, 2018), y en particular (Langheinrich, 2018) al centrarse en el factor de impacto como una métrica de "popularidad" que no distingue entre las fuentes de citas: una cita de un artículo aparecido en una revista muy bien indexada cuenta tanto como una en una revista menor. La crítica viene por contar las "autocitas", citas de un artículo de una revista a otros artículos de la misma revista, algo muchas veces promovido por editores con mala praxis que condicionan la aceptación del artículo a la cita de artículos de su cabecera, incrementando de forma artificial su número. Esto provoca que en la WoS se supervisen las autocitas de publicaciones y se creen listas negras de revistas que se autocitan en exceso o intentan hacer trampa con el Factor de Impacto ${ }^{1}$. Cabe recordar con (Chinchilla-Rodríguez, 2018) que en sí, no es sinónimo de evaluación científica, pues no deja de ser una herramienta de la que se puede hacer un buen o mal uso.

En particular nos centraremos en Twitter como herramienta docente, por el interés general despertado en la enseñanza superior (Kassens-Noor, 2012), (Veletsianos, 2012), (Guzmán Duque, Del Moral Pérez, \& González Ladrón de Guevara, 2012), (Alonso García \& Alonso García, 2014) y por nuestro propio bagaje, como usuarios de la misma que practican un uso docente con ella.

\footnotetext{
${ }^{1}$ Listado disponible en http://ipscience-help.thomsonreuters.com/incitesLiveJCR/JCRGroup/titleSuppressions.html
} 


\section{Estudio}

La información presente se recopiló de la (WoS), la base de datos más influyente en ámbitos académicos, evaluando la información desde diferentes perspectivas: países, autores y artículos más citados. Básicamente, el enfoque dado es el clásico en el análisis de citas: número de publicaciones y h- index.

Sobre este índice cabe detenernos unos instantes. Si el número de artículos indica productividad y el número de citas indica influencia en un área de investigación, el h- índex representa una combinación de ambos.

En el presente trabajo analizamos los artículos aparecidos en el periodo 2009-2017, clasificando los mismos por países (los 50 con más citas), las organizaciones (universidades), autores y artículos más citados, presentando la información a través de tablas que consideran la cantidad total de documentos (TS), el total de citas (TC) y el híndex. Esto nos da pie para hablar del factor de impacto (Langheinrich, 2018) de un año (o serie de años) determinado(s) que podría ser la que nos da el número de veces que un artículo publicado fue citado en cualquiera de las publicaciones incluidas en la base de datos consultada, dividido por el número total de artículos que citan.

Vamos, sin más dilación, a mostrar los resultados obtenidos.

En primer lugar presentamos un listado de los 50 autores principales, con indicación de su indicación y país, en la tabla siguiente.

Tabla 1. Principales autores. Fuente: Elaboración propia.

\begin{tabular}{|l|l|l|l|l|l|l|l|}
\hline $\mathbf{R}$ & Name & University & Country & TS & TC & H & TC/TS \\
\hline 1 & Carpenter, Jeffrey & Middlebury College & USA & 7 & 24 & 3 & 3,43 \\
\hline 2 & Chan, Teresa M & McMaster University & Canada & 6 & 36 & 6 & 6,00 \\
\hline 3 & Lin, Michelle & McMaster University & Canada & 5 & 54 & 4 & 10,80 \\
\hline 4 & Thoma, Brent & University of Saskatchewan & Canada & 5 & 36 & 2 & 7,20 \\
\hline 5 & Veletsianos, George & Royal Roads University, & Canada & 5 & 90 & 3 & 18,00 \\
\hline 6 & Gardner, Jerad M & University of Arkansas for Medical & USA & 4 & 4 & 1 & 1,00 \\
\hline 7 & Luo, Tian & Ohio University & USA & 4 & 66 & 2 & 16,50 \\
\hline 8 & Allem, Jon-Patrick & University of Southern California & USA & 3 & 13 & 2 & 4,33 \\
\hline 9 & Attai, Deanna J & University of California Los Angeles & USA & 3 & 38 & 1 & 12,67 \\
\hline 10 & Braddy, Richard & Western General Hospital & UK & 3 & 17 & 1 & 5,67 \\
\hline 11 & Chu, Kar-Hai & University of Pittsburgh & USA & 3 & 13 & 2 & 4,33 \\
\hline 12 & Cooney, Robert & Geisinger Medical Center & USA & 3 & 9 & 1 & 3,00 \\
\hline 13 & Cruz, Tess Boley & University of Southern California & USA & 3 & 13 & 2 & 4,33 \\
\hline 14 & Fox, Alison & University of Leicester & UK & 3 & 1 & 1 & 0,33 \\
\hline 15 & Gottlieb, Michael & Rush University Medical Center, & USA & 3 & 0 & 0 & 0,00 \\
\hline 16 & Junco, Reynol & Lock Haven Univ. & USA & 3 & 385 & 3 & 128,33 \\
\hline 17 & Keengwe, J & University of North Dakota & USA & 3 & 0 & 0 & 0,00 \\
\hline 18 & Kimmons, Royce & Bringham Young University & USA & 3 & 14 & 2 & 4,67 \\
\hline 19 & Lemon, Narelle & La Trobe Univ, & Australia & 3 & 9 & 2 & 3,00 \\
\hline 20 & Leveridge, Michael J & Queen's University & Canada & 3 & 60 & 2 & 20,00 \\
\hline 21 & Manecksha, R. P & Tallaght Hospital & Ireland & 3 & 50 & 2 & 16,67 \\
\hline 22 & Marin, Victoria I. & University of the Balearic Islands & Spain & 3 & 10 & 2 & 3,33 \\
\hline 23 & Merchant, Raina M. & University of Pennsylvania & USA & 3 & 82 & 2 & 27,33 \\
\hline 24 & Nason, Gregory J & University Hospital Limerick & Ireland & 3 & 50 & 2 & 16,67 \\
\hline 25 & O'Kelly, Fardod & University Hospital Limerick & Ireland & 3 & 50 & 2 & 16,67 \\
\hline
\end{tabular}




\begin{tabular}{|l|l|l|l|l|l|l|l|}
\hline $\mathbf{R}$ & Name & University & Country & TS & TC & H & TC/TS \\
\hline 26 & Sherbino, Jonathan & McMaster University & Canada & 3 & 22 & 2 & 7,33 \\
\hline 27 & Thompson, Michael A & Aurora Research Institute, & USA & 3 & 22 & 2 & 7,33 \\
\hline 28 & Tur, Gemma & University of the Balearic Islands & Spain & 3 & 10 & 2 & 3,33 \\
\hline 29 & Unger, Jennifer B. & University of Southern California & USA & 3 & 13 & 2 & 4,33 \\
\hline 30 & Watson, Jason & Foothills Medical Centre & Canada & 3 & 13 & 1 & 4,33 \\
\hline 31 & Al-Bahrani, Abdullah & Northern Kentucky University & USA & 2 & 20 & 2 & 10,00 \\
\hline 32 & Alotaibi, Naif M & University of Toronto & Canada & 2 & 12 & 2 & 6,00 \\
\hline 33 & Aschc, David A. & University of Pennsylvania & USA & 2 & 23 & 1 & 11,50 \\
\hline 34 & Bachrach, Yoram & Microsoft Research & UK & 2 & 19 & 2 & 9,50 \\
\hline 35 & Beardmore, C. & Society and College of Radiographers & UK & 2 & 0 & 0 & 0,00 \\
\hline 36 & Bernhardt, Jay M. & University of Texas & USA & 2 & 33 & 2 & 16,50 \\
\hline 37 & Bird, Terese & University of Leicester & UK & 2 & 1 & 1 & 0,50 \\
\hline 38 & Bolderston, A & British Columbia Cancer Agency & Canada & 2 & 0 & 0 & 0,00 \\
\hline 39 & Cabrera, Daniel & Mavo Clinic & USA & 2 & 3 & 1 & 1,50 \\
\hline 40 & Chisolm, Margaret S. & Johns Hopkins University & USA & 2 & 5 & 1 & 2,50 \\
\hline 41 & Cumming, Grant P. & Dr Gray's Hospital & UK & 2 & 44 & 1 & 22,00 \\
\hline 42 & Currie, Geoff & University of Toronto & Canada & 2 & 0 & 0 & 0,00 \\
\hline 43 & Dai, Hongying & University of Missouri-Kansas City & USA & 2 & 4 & 1 & 2,00 \\
\hline 44 & Di Prospero, L. & University of Toronto & Canada & 2 & 0 & 0 & 0,00 \\
\hline 45 & Djuricich, Alexander & Indiana University & USA & 2 & 20 & 2 & 10,00 \\
\hline 46 & Duszak, Richard & Emory University School of & USA & 2 & 74 & 2 & 37,00 \\
\hline 47 & Ebner, M & Computer and Information Services & Austria & 2 & 172 & 1 & 86,00 \\
\hline 48 & Escobedo, Patricia & University of Southern California & USA & 2 & 4 & 1 & 2,00 \\
\hline 49 & Fernandez-Diaz, Elia & University of Cantabria & Spain & 2 & 13 & 1 & 6,50 \\
\hline 50 & Fouz-Gonzalez, Jonas & Universidad Católica de Murcia & Spain & 2 & 5 & 1 & 2,50 \\
\hline
\end{tabular}

En la siguiente tabla podemos ver una clasificación por las organziaciones de procedencia de los autores.

Tabla 2. Principales instituciones. Fuente: Elaboración propia.

\begin{tabular}{|c|c|c|c|c|c|c|c|c|c|c|c|}
\hline $\mathbf{R}$ & Institution & Country & TS & TC & $\mathbf{H}$ & $\begin{array}{l}\text { TC/ } \\
\text { TS }\end{array}$ & $\begin{array}{l}>= \\
100\end{array}$ & $\begin{array}{l}>= \\
\mathbf{5 0}\end{array}$ & $\begin{array}{l}>= \\
20\end{array}$ & $\begin{array}{l}\mathbf{A R} \\
\mathbf{W U} \\
\end{array}$ & QS \\
\hline 1 & University of Toronto & Canada & 14 & 141 & 6 & 10,0 & 0 & 1 & 2 & 23 & 31 \\
\hline 2 & Harvard University & USA & 12 & 92 & 5 & 7,67 & 0 & 0 & 3 & 1 & 3 \\
\hline 3 & University of Ottawa & Canada & 11 & 72 & 6 & 6,55 & 0 & 0 & 0 & $141-$ & 289 \\
\hline 4 & McMaster University & Canada & 10 & 52 & 4 & 5,20 & 0 & 0 & 1 & 66 & 140 \\
\hline 5 & CLEVELAND CLINIC & USA & 9 & 25 & 3 & 2,78 & 0 & 0 & 0 & & N/A \\
\hline 6 & JOHNS HOPKINS UNIVERSITY & USA & 9 & 43 & 5 & 4,78 & 0 & 0 & 0 & 18 & 17 \\
\hline 7 & UNIVERSITY OF CALIFORNIA & USA & 9 & 80 & 5 & 8,89 & 0 & 0 & 2 & 21 & 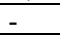 \\
\hline 8 & UNIVERSITY OF KENTUCKY & USA & 8 & 43 & 4 & 5,38 & 0 & 0 & 1 & $301-$ & $551-$ \\
\hline 9 & UNIVERSITY OF MICHIGAN & USA & 8 & 110 & 5 & 13,7 & 0 & 0 & 3 & 24 & 21 \\
\hline 10 & UNIVERSITY OF & USA & 8 & 93 & 3 & 11,6 & 0 & 1 & 1 & 17 & 19 \\
\hline 11 & UNIVERSITY OF MARYLAND & USA & 7 & 57 & 4 & 8,14 & 0 & 0 & 1 & $201-$ & 481- \\
\hline 12 & DUKE UNIVERSITY & USA & 6 & 28 & 3 & 4,67 & 0 & 0 & 1 & 26 & 21 \\
\hline 13 & ELON UNIVERSITY & USA & 6 & 17 & 2 & 2,83 & 0 & 0 & 0 & 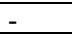 & . \\
\hline 14 & UNIVERSITY COLLEGE & UK & 6 & 51 & 4 & 8,50 & 0 & 0 & 1 & 16 & 7 \\
\hline 15 & UNIVERSITY OF ARKANSAS & USA & 6 & 31 & 2 & 5,17 & 0 & 0 & 1 & - & 801- \\
\hline 16 & UNIVERSITY OF CALIFORNIA & USA & 6 & 46 & 3 & 7,67 & 0 & 0 & 1 & 12 & 33 \\
\hline 17 & UNIVERSITY OF FLORIDA & USA & 6 & 80 & 2 & 13,3 & 0 & 1 & 2 & 88 & 178 \\
\hline 18 & UNIVERSITY OF MARYLAND & USA & 6 & 4 & 2 & 0,67 & 0 & 0 & 0 & 53 & 129 \\
\hline 19 & UNIVERSITY OF SYDNEY & AUSTR & 6 & 113 & 5 & 18,8 & 0 & 1 & 1 & 83 & 50 \\
\hline 20 & MAYO CLINIC & USA & 5 & 6 & 2 & 1,20 & 0 & 0 & 0 & 71 & 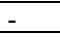 \\
\hline 21 & NATIONAL INSTITUTES OF & USA & 5 & 43 & 4 & 8,60 & 0 & 0 & 0 & & \\
\hline 22 & NEW YORK UNIVERSITY & USA & 5 & 40 & 3 & 8,00 & 0 & 0 & 1 & 29 & 52 \\
\hline 23 & OHIO STATE UNIVERSITY & USA & 5 & 37 & 2 & 7,40 & 0 & 0 & 1 & 80 & 86 \\
\hline 24 & OPEN UNIVERSITY UK & UK & 5 & 35 & 2 & 7,00 & 0 & 0 & 1 & & \\
\hline 25 & UNIVERSITY OF GRANADA & SPAIN & 5 & 9 & 1 & 1,80 & 0 & 0 & 0 & $201-$ & $501-$ \\
\hline 26 & $\begin{array}{l}\text { UNIVERSITY OF LEICESTER } \\
\end{array}$ & UK & 5 & 8 & 2 & 1,60 & 0 & 0 & 0 & $301-$ & 238 \\
\hline
\end{tabular}




\begin{tabular}{|l|l|l|l|l|l|l|l|l|l|l|l|}
\hline $\mathbf{R}$ & Institution & $\mathbf{C o u n t r y}$ & $\mathbf{T S}$ & $\mathbf{T C}$ & $\mathbf{H}$ & $\begin{array}{l}\mathbf{T C} / \\
\mathbf{T S}\end{array}$ & $\begin{array}{l}>= \\
\mathbf{1 0 0}\end{array}$ & $\begin{array}{l}>= \\
\mathbf{5 0}\end{array}$ & $\begin{array}{l}>= \\
\mathbf{2 0}\end{array}$ & $\begin{array}{l}\text { AR } \\
\mathbf{W U}\end{array}$ & $\begin{array}{l}\text { QS } \\
\end{array}$ \\
\hline 27 & UNIVERSITY OF MELBOURNE & AUSTR & 5 & 174 & 5 & 34,8 & 0 & 1 & 4 & 39 & 41 \\
\hline 28 & UNIVERSITY OF MINNESOTA & USA & 5 & 178 & 4 & 35,6 & 1 & 1 & 2 & 34 & 163 \\
\hline 29 & UNIVERSITY OF NORTH & USA & 5 & 40 & 4 & 8,00 & 0 & 0 & 0 & 33 & 80 \\
\hline 30 & UNIVERSITY OF NOTTINGHAM & UK & 5 & 1 & 1 & 0,20 & 0 & 0 & 0 & $101-$ & 84 \\
\hline 31 & UNIVERSITY OF PITTSBURGH & USA & 5 & 85 & 2 & 17,0 & 0 & 1 & 2 & 68 & 142 \\
\hline 32 & UNIVERSITY OF & CANAD & 5 & 35 & 2 & 7,00 & 0 & 0 & 1 & $301-$ & $451-$ \\
\hline 33 & UNIVERSITY OF WASHINGTON & USA & 5 & 117 & 2 & 23,4 & 0 & 1 & 2 & 13 & 61 \\
\hline 34 & BAYLOR COLLEGE OF & USA & 4 & 11 & 2 & 2,75 & 0 & 0 & 0 & $101-$ & $801-$ \\
\hline 35 & GEORGETOWN UNIVERSITY & USA & 4 & 41 & 3 & 10,2 & 0 & 0 & 0 & $201-$ & 227 \\
\hline 36 & GRIFFITH UNIVERSITY & AUSTR & 4 & 42 & 3 & 10,5 & 0 & 0 & 1 & $301-$ & 325 \\
\hline 37 & INDIANA UNIVERSITY & USA & 4 & 13 & 2 & 3,25 & 0 & 0 & 0 & $101-$ & 304 \\
\hline 38 & MEDEDLIFE RES COLLABORAT & USA & 4 & 31 & 2 & 7,75 & 0 & 0 & 1 & - & - \\
\hline 39 & MONASH UNIVERSITY & AUSTR & 4 & 7 & 2 & 1,75 & 0 & 0 & 0 & 78 & 60 \\
\hline 40 & PURDUE UNIVERSITY & USA & 4 & 90 & 4 & 22,5 & 0 & 0 & 3 & 77 & 105 \\
\hline 41 & QUEENS UNIVERSITY CANADA & CANAD & 4 & 122 & 3 & 30,5 & 0 & 1 & 2 & - & - \\
\hline 42 & RESEARCH TRIANGLE & USA & 4 & 45 & 2 & 11,2 & 0 & 0 & 1 & - & - \\
\hline 43 & RUSH UNIVERSITY & USA & 4 & 0 & 0 & 0,00 & 0 & 0 & 0 & $401-$ & - \\
\hline 44 & UNIVERSITAT DE LES ILLES & SPAIN & 4 & 33 & 2 & 8,25 & 0 & 0 & 1 & - & - \\
\hline 45 & UNIVERSITY OF CHICAGO & USA & 4 & 16 & 2 & 4,00 & 0 & 0 & 0 & 10 & 9 \\
\hline 46 & UNIVERSITY OF EDINBURGH & UK & 4 & 25 & 2 & 6,25 & 0 & 0 & 0 & 32 & 23 \\
\hline 47 & UNIVERSITY OF HONG KONG & HONG & 4 & 41 & 3 & 10,2 & 0 & 0 & 1 & $101-$ & 26 \\
\hline 48 & UNIVERSITY OF ILLINOIS & USA & 4 & 9 & 2 & 2,25 & 0 & 0 & 0 & 37 & 69 \\
\hline 49 & UNIVERSITY OF MISSOURI & USA & 4 & 7 & 2 & 1,75 & 0 & 0 & 0 & $201-$ & $601-$ \\
\hline 50 & UNIVERSITY OF PLYMOUTH & UK & 4 & 23 & 3 & 5,75 & 0 & 0 & 0 & - & $701-$ \\
\hline
\end{tabular}

Antes de proceder a crear las tablas de resumen, una última tabla donde ordenamos los 50 artículos más citados, con indicación del año y un índice donde se relacionan las citas por los años transcurridos.

Tabla 3. Artículos más citados. Fuente: Elaboración propia.

\begin{tabular}{|c|c|c|c|c|c|}
\hline $\mathbf{R}$ & TC & Title & Authors & Year & Cit/year \\
\hline 1 & 291 & $\begin{array}{l}\text { The effect of Twitter on college student } \\
\text { engagement and grades }\end{array}$ & $\begin{array}{ll}\text { Junco, } & \text { R.; } \\
\text { Heiberger, } & \text { G.; } \\
\text { Loken, E. } & \\
\end{array}$ & 2011 & 41,57142857 \\
\hline 2 & 229 & $\begin{array}{l}\text { Dissemination of health information through social } \\
\text { networks: Twitter and antibiotics }\end{array}$ & $\begin{array}{l}\text { Scanfeld, Daniel; } \\
\text { Scanfeld, } \\
\text { Vanessa; Larson, } \\
\text { Elaine L. }\end{array}$ & 2010 & 28,625 \\
\hline 3 & 174 & $\begin{array}{l}\text { Microblogs in Higher Education - A chance to } \\
\text { facilitate informal and process-oriented learning? }\end{array}$ & $\begin{array}{l}\text { Ebner, Martin; } \\
\text { Lienhardt, } \\
\text { Conrad; Rohs, } \\
\text { Matthias; et ál.. }\end{array}$ & 2010 & 21,75 \\
\hline 4 & 141 & $\begin{array}{l}\text { The role of social media in higher education classes } \\
\text { (real and virtual) - A literature review }\end{array}$ & Tess, Paul A. & 2013 & 28,2 \\
\hline 5 & 87 & $\begin{array}{l}\text { The Geography of Happiness: Connecting Twitter } \\
\text { Sentiment and Expression, Demographics, and } \\
\text { Objective Characteristics of Place }\end{array}$ & $\begin{array}{l}\text { Mitchell, Lewis; } \\
\text { Frank, Morgan R.; } \\
\text { Harris, Kameron } \\
\text { Decker; et ál.. }\end{array}$ & 2013 & 17,4 \\
\hline 6 & 76 & $\begin{array}{l}\text { Using Twitter to Examine Smoking Behavior and } \\
\text { Perceptions of Emerging Tobacco Products }\end{array}$ & $\begin{array}{l}\text { Myslin, Mark; } \\
\text { Zhu, Shu-Hong; } \\
\text { Chapman, Wendy; } \\
\text { et ál.. }\end{array}$ & 2013 & 15,2 \\
\hline 7 & 76 & $\begin{array}{l}\text { Higher education scholars' participation and } \\
\text { practices on Twitter }\end{array}$ & Veletsianos, G. & 2012 & 12,66666667 \\
\hline
\end{tabular}




\begin{tabular}{|c|c|c|c|c|c|}
\hline 8 & 73 & $\begin{array}{l}\text { Putting twitter to the test: Assessing outcomes for } \\
\text { student collaboration, engagement and success }\end{array}$ & $\begin{array}{lr}\text { Junco, } & \text { Reynol; } \\
\text { Elavsky, } & \text { C. } \\
\text { Michael; } & \\
\text { Heiberger, } & \text { Greg }\end{array}$ & 2013 & 14,6 \\
\hline 9 & 72 & $\begin{array}{l}\text { Twitter as a teaching practice to enhance active and } \\
\text { informal learning in higher education: The case of } \\
\text { sustainable tweets }\end{array}$ & $\begin{array}{l}\text { Kassens-Noor, } \\
\text { Eva }\end{array}$ & 2012 & 12 \\
\hline 10 & 66 & $\begin{array}{l}\text { Research Blogs and the Discussion of Scholarly } \\
\text { Information }\end{array}$ & $\begin{array}{l}\text { Shema, Hadas; } \\
\text { Bar-Ilan, Judit; } \\
\text { Thelwall, }\end{array}$ & 2012 & 11 \\
\hline 11 & 64 & $\begin{array}{l}\text { International Urology Journal Club via Twitter: 12- } \\
\text { Month Experience }\end{array}$ & $\begin{array}{l}\text { Thangasamy, } \\
\text { Isaac A.; } \\
\text { Leveridge, } \\
\text { Michael; Davies, } \\
\text { Benjamin J.; et ál.. }\end{array}$ & 2014 & 16 \\
\hline 12 & 63 & $\begin{array}{l}\text { Tweeting for learning: A critical analysis of } \\
\text { research on microblogging in education published } \\
\text { in 2008-2011 }\end{array}$ & $\begin{array}{l}\text { Gao, Fei; Luo, } \\
\text { Tian; Zhang, Ke }\end{array}$ & 2012 & 10,5 \\
\hline 13 & 60 & $\begin{array}{l}\text { Psychological Language on } \quad \text { Twitter } \\
\text { County-Level Heart Disease Mortality }\end{array}$ & $\begin{array}{l}\text { Eichstaedt, } \\
\text { Johannes C.; } \\
\text { Schwartz, Hansen } \\
\text { Andrew; Kern, } \\
\text { Margaret L.; et ál.. }\end{array}$ & 2015 & 20 \\
\hline 14 & 55 & $\begin{array}{l}\text { A case study of Israeli higher-education institutes } \\
\text { sharing scholarly information with the community } \\
\text { via social networks }\end{array}$ & $\begin{array}{l}\text { Forkosh-Baruch, } \\
\text { Alona; } \\
\text { Hershkovitz, } \\
\text { Arnon }\end{array}$ & 2012 & 9,166666667 \\
\hline 15 & 53 & $\begin{array}{l}\text { eHealth Literacy and Web } 2.0 \text { Health Information } \\
\text { Seeking Behaviors Among Baby Boomers and } \\
\text { Older Adults }\end{array}$ & $\begin{array}{l}\text { Tennant, Bethany; } \\
\text { Stellefson, } \\
\text { Michael; Dodd, } \\
\text { Virginia; et ál.. }\end{array}$ & 2015 & 17,66666667 \\
\hline 16 & 49 & $\begin{array}{l}\text { Use of Web } 2.0 \text { technologies in K-12 and higher } \\
\text { education: The search for evidence-based practice }\end{array}$ & $\begin{array}{ll}\text { Hew, Khe } & \text { Foon; } \\
\text { Cheung, } & \text { Wing } \\
\text { Sum } & \end{array}$ & 2013 & 9,8 \\
\hline 17 & 49 & $\begin{array}{l}\text { 'What's happening?' A content analysis of } \\
\text { concussion-related traffic on Twitter }\end{array}$ & $\begin{array}{l}\text { Sullivan, S. John; } \\
\text { Schneiders, } \\
\text { Anthony G.; } \\
\text { Cheang, Choon- } \\
\text { Wi; et ál.. }\end{array}$ & 2012 & 8,166666667 \\
\hline 18 & 48 & $\begin{array}{l}\text { Social Media, Text Messaging, and Email- } \\
\text { Preferences of Asthma Patients between } 12 \text { and } 40 \\
\text { Years Old }\end{array}$ & $\begin{array}{l}\text { Baptist, Alan P.; } \\
\text { Thompson, } \\
\text { Michael; } \\
\text { Grossman, Karla } \\
\text { Stoermer; et ál.. }\end{array}$ & 2011 & 6,857142857 \\
\hline 19 & 46 & $\begin{array}{l}\text { Social Media in Radiology: Early Trends in Twitter } \\
\text { Microblogging at Radiology's Largest International } \\
\text { Meeting }\end{array}$ & $\begin{array}{l}\text { Hawkins, C. } \\
\text { Matthew; Duszak, } \\
\text { Richard; Rawson, } \\
\text { James V. }\end{array}$ & 2014 & 11,5 \\
\hline 20 & 44 & $\begin{array}{l}\text { Increased Use of Twitter at a Medical Conference: } \\
\text { A Report and a Review of the Educational } \\
\text { Opportunities }\end{array}$ & $\begin{array}{l}\text { McKendrick, } \\
\text { Douglas R. A.; } \\
\text { Cumming, Grant } \\
\text { P.; Lee, Amanda } \\
\text { J. }\end{array}$ & 2012 & 7,333333333 \\
\hline 21 & 43 & The Dramatic Increase in Social Media in Urology & $\begin{array}{ll}\text { Matta, } & \text { Rano; } \\
\text { Doiron, } & \text { Chris; } \\
\text { Leveridge, } & \\
\text { Michael J. } & \end{array}$ & 2014 & 10,75 \\
\hline
\end{tabular}




\begin{tabular}{|c|c|c|c|c|c|}
\hline 22 & 40 & $\begin{array}{l}\text { Use of social media in graduate-level medical } \\
\text { humanities education: Two pilot studies from Penn } \\
\text { State College of Medicine }\end{array}$ & 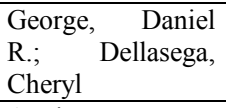 & 2011 & 5,714285714 \\
\hline 23 & 39 & $\begin{array}{l}\text { Twitter Social Media is an Effective Tool for } \\
\text { Breast Cancer Patient Education and Support: } \\
\text { Patient-Reported Outcomes by Survey }\end{array}$ & $\begin{array}{l}\text { Attai, Deanna J.; } \\
\text { Cowher, Michael } \\
\text { S.; Al-Hamadani, } \\
\text { Mohammed; et ál. }\end{array}$ & 2015 & 13 \\
\hline 24 & 37 & The emerging use of Twitter by urological journals & $\begin{array}{lr}\text { Nason, } & \text { Gregory } \\
\text { J.; } & \text { O'Kelly, } \\
\text { Fardod; } & \text { Kelly, } \\
\text { Michael E.; et ál.. }\end{array}$ & 2015 & 12,33333333 \\
\hline 25 & 36 & $\begin{array}{l}\text { Social media adoption at the American grass roots: } \\
\text { Web } 2.0 \text { or } 1.5 \text { ? }\end{array}$ & $\begin{array}{l}\text { Reddick, } \\
\text { Christopher G.; } \\
\text { Norris, Donald F. }\end{array}$ & 2013 & 7,2 \\
\hline 26 & 32 & $\begin{array}{l}\text { The use of social-networking sites in medical } \\
\text { education }\end{array}$ & $\begin{array}{l}\text { Cartledge, Peter; } \\
\text { Miller, Michael; } \\
\text { Phillips, Bob }\end{array}$ & 2013 & 6,4 \\
\hline 27 & 31 & $\begin{array}{l}\text { Museum learning via social and mobile } \\
\text { technologies: (How) can online interactions } \\
\text { enhance the visitor experience? }\end{array}$ & $\begin{array}{l}\text { Charitonos, } \\
\text { Koula; Blake, } \\
\text { Canan; Scanlon, } \\
\text { Eileen; et ál.. }\end{array}$ & 2012 & 5,166666667 \\
\hline 28 & 30 & Social networking tools for academic libraries & $\begin{array}{l}\text { Chu, Samuel Kai- } \\
\text { Wah; Du, Helen } \\
\text { S. }\end{array}$ & 2013 & 6 \\
\hline 29 & 30 & $\begin{array}{l}\text { Guidelines for Maintaining a Professional Compass } \\
\text { in the Era of Social Networking }\end{array}$ & $\begin{array}{l}\text { Landman, } \\
\text { Matthew P.; } \\
\text { Shelton, Julia; } \\
\text { Kauffmann, Rondi } \\
\text { M.; et ál. }\end{array}$ & 2010 & 3,75 \\
\hline 30 & 29 & $\begin{array}{l}\text { Are Public Health Organizations Tweeting to the } \\
\text { Choir? Understanding Local Health Department } \\
\text { Twitter Followership }\end{array}$ & $\begin{array}{l}\text { Harris, Jenine K.; } \\
\text { Choucair, } \\
\text { Bechara; Maier, } \\
\text { Ryan C.; et ál.. }\end{array}$ & 2014 & 7,25 \\
\hline 31 & 28 & $\begin{array}{l}\text { The Impact of Social Media on Readership of a } \\
\text { Peer-Reviewed Medical Journal }\end{array}$ & $\begin{array}{l}\text { Hawkins, C. } \\
\text { Matthew; } \\
\text { Hillman, Bruce J.; } \\
\text { Carlos, Ruth C.; et } \\
\text { ál.. }\end{array}$ & 2014 & 7 \\
\hline 32 & 28 & $\begin{array}{l}\text { Twitter for teaching: Can social media be used to } \\
\text { enhance the process of learning? }\end{array}$ & Evans, Chris & 2014 & 7 \\
\hline 33 & 28 & Inequalities in Facebook use & Junco, Reynol & 2013 & 5,6 \\
\hline 34 & 28 & Digital Social Networks and Health & $\begin{array}{l}\text { Lefebvre, } \quad \text { R. } \\
\text { Craig; Bornkessel, } \\
\text { Alexandra S. }\end{array}$ & 2013 & 5,6 \\
\hline 35 & 27 & $\begin{array}{lccc}\text { Social Media and Internet Driven Study } \\
\text { Recruitment: Evaluating a New Model for } \\
\text { Promoting } & \text { Collaborator } & \text { Engagement } & \text { and } \\
\text { Participation } & & & \end{array}$ & $\begin{array}{l}\text { Khatri, Chetan; } \\
\text { Chapman, } \\
\text { Stephen J.; } \\
\text { Glasbey, James; et } \\
\text { ál.. }\end{array}$ & 2015 & 9 \\
\hline 36 & 26 & $\begin{array}{l}\text { Globalization of Continuing Professional } \\
\text { Development by Journal Clubs via Microblogging: } \\
\text { A Systematic Review }\end{array}$ & $\begin{array}{l}\text { Roberts, Matthew } \\
\text { John; Perera, } \\
\text { Marlon; } \\
\text { Lawrentschuk, } \\
\text { Nathan; et ál.. } \\
\end{array}$ & 2015 & 8,666666667 \\
\hline 37 & 26 & $\begin{array}{l}\text { Exploring the Use of a Facebook Page in Anatomy } \\
\text { Education }\end{array}$ & $\begin{array}{ll}\text { Jaffar, } & \text { Akram } \\
\text { Abood } & \\
\end{array}$ & 2014 & 6,5 \\
\hline
\end{tabular}




\begin{tabular}{|c|c|c|c|c|c|}
\hline 38 & 26 & $\begin{array}{l}\text { Construction of the Foundations of the PLE and } \\
\text { PLN for Collaborative Learning }\end{array}$ & $\begin{array}{l}\text { Marin, Victoria; } \\
\text { Negre, Francisca; } \\
\text { Perez, Adolfina }\end{array}$ & 2014 & 6,5 \\
\hline 39 & 25 & $\begin{array}{l}\text { Investigating the Community of Practice of World } \\
\text { Language Educators on Twitter }\end{array}$ & $\begin{array}{l}\text { Wesely, Pamela } \\
\text { M. }\end{array}$ & 2013 & 5 \\
\hline 40 & 24 & $\begin{array}{l}\text { The Social Media Index: Measuring the Impact of } \\
\text { Emergency Medicine and Critical Care Websites }\end{array}$ & $\begin{array}{l}\text { Thoma, Brent; } \\
\text { Sanders, Jason L.; } \\
\text { Lin, Michelle; et } \\
\text { ál.. }\end{array}$ & 2015 & 8 \\
\hline 41 & 24 & $\begin{array}{l}\text { Australian health professions student use of social } \\
\text { media }\end{array}$ & $\begin{array}{lr}\text { Usher, } & \text { Kim; } \\
\text { Woods, Cindy; } \\
\text { Casella, Evan; et } \\
\text { ál.. }\end{array}$ & 2014 & 6 \\
\hline 42 & 24 & Social media and the introductory statistics course & $\begin{array}{l}\text { Everson, } \\
\text { Michelle; } \\
\text { Gundlach, Ellen; } \\
\text { Miller, Jacqueline }\end{array}$ & 2013 & 4,8 \\
\hline 43 & 23 & $\begin{array}{l}\text { Social Media Use in Chronic Disease: A } \\
\text { Systematic Review and Novel Taxonomy }\end{array}$ & $\begin{array}{l}\text { Patel, Rajesh; } \\
\text { Chang, Tammy; } \\
\text { Greysen, S. Ryan; } \\
\text { et ál.. }\end{array}$ & 2015 & 7,666666667 \\
\hline 44 & 23 & $\begin{array}{l}\text { Decoding twitter: Surveillance and trends for } \\
\text { cardiac arrest and resuscitation communication }\end{array}$ & $\begin{array}{l}\text { Bosley, Justin C.; } \\
\text { Zhao, Nina W.; } \\
\text { Hill, Shawndra; et } \\
\text { ál.. }\end{array}$ & 2013 & 4,6 \\
\hline 45 & 22 & $\begin{array}{l}\text { Facebook and the others. Potentials and obstacles } \\
\text { of Social Media for teaching in higher education }\end{array}$ & $\begin{array}{l}\text { Manca, Stefania; } \\
\text { Ranieri, Maria }\end{array}$ & 2016 & 11 \\
\hline 46 & 22 & $\begin{array}{l}\text { Mobile Learning with Twitter to Improve } \\
\text { Linguistic Competence at Secondary Schools }\end{array}$ & $\begin{array}{l}\text { Bosley, Justin C.; } \\
\text { Zhao, Nina W.; } \\
\text { Hill, Shawndra; et } \\
\text { ál.. }\end{array}$ & 2012 & 3,666666667 \\
\hline 47 & 21 & The Role of Social Media in Dental Education & $\begin{array}{l}\text { McAndrew, } \\
\text { Maureen; } \\
\text { Johnston, Amelia } \\
\text { E. }\end{array}$ & 2012 & 3,5 \\
\hline 48 & 21 & Use of Social Media by Pharmacy Preceptors & $\begin{array}{lr}\text { Kukreja, } & \text { Pavnit; } \\
\text { Sheehan, } & \text { Amy } \\
\text { Heck; } & \text { Riggins, } \\
\text { Jennifer } & \end{array}$ & 2011 & 3 \\
\hline 49 & 20 & $\begin{array}{l}\text { Using Social Media to Share Your Radiology } \\
\text { Research: How Effective Is a Blog Post? }\end{array}$ & $\begin{array}{l}\text { Hoang, Jenny K.; } \\
\text { McCall, Jonathan; } \\
\text { Dixon, Andrew } \\
\text { F.; et ál.. }\end{array}$ & 2015 & 6,666666667 \\
\hline 50 & 20 & Obesity in social media: a mixed methods analysis & $\begin{array}{lr}\text { Chou, } & \text { Wen-ying } \\
\text { Sylvia; } & \text { Prestin, } \\
\text { Abby; } & \text { Kunath, } \\
\text { Stephen } & \end{array}$ & 2014 & 5 \\
\hline
\end{tabular}




\section{Resultados}

Analizados los datos precedentes, observamos que mientras los Estados Unidos de América, Gran Bretaña y Canadá ocupan los primeros lugares, España se configura como candidato a figurar entre los países que más producción científica dedican al estudio de Twitter en la educación. Esto, considerando los tamaños poblacionales de los distintos países, es algo más que notorio. En la tabla siguiente podemos ver un resumen de los datos, que nos permiten alcanzar esta idea:

Tabla 4. Resumen de citas por países para el periodo estudiado. Fuente: Elaboración propia.

\begin{tabular}{|l|l|l|l|l|l|l|l|l|l|l|}
\hline Year & USA & UK & Canada & Spain & Australia & China & Saudi & Malavsia & Germanv & Ireland \\
\hline 2009 & 1 & 0 & 0 & 0 & 0 & 0 & 0 & 0 & 0 & 0 \\
\hline 2010 & 8 & 0 & 0 & 0 & 1 & 0 & 0 & 0 & 0 & 0 \\
\hline 2011 & 12 & 1 & 1 & 0 & 0 & 0 & 0 & 0 & 0 & 0 \\
\hline 2012 & 8 & 3 & 2 & 1 & 2 & 1 & 1 & 0 & 0 & 0 \\
\hline 2013 & 29 & 3 & 2 & 3 & 3 & 2 & 1 & 0 & 1 & 0 \\
\hline 2014 & 21 & 6 & 3 & 3 & 6 & 0 & 0 & 2 & 0 & 1 \\
\hline 2015 & 51 & 12 & 10 & 8 & 12 & 2 & 1 & 1 & 1 & 3 \\
\hline 2016 & 53 & 18 & 12 & 13 & 9 & 3 & 2 & 2 & 2 & 1 \\
\hline 2017 & 76 & 28 & 17 & 15 & 7 & 5 & 4 & 2 & 2 & 1 \\
\hline
\end{tabular}

\section{Conclusiones}

Observamos que un gran número de artículos se centran en la docencia del área de salud. Esto nos hace coincidir con las apreciaciones de (Dorta-Contreras, 2018). De hecho, de los 635 artículos estudiados, un 37\% corresponden a esa área, siendo el área de educación (en general, formación de formadores o sin establecer como elemento distintivo el tipo de estudios de los discentes) un 30\%. Un área donde se podría esperar a prioir un gran impacto, por la elevada alfabetización tecnológica de estudiantes y docentes, el área de las TC, tan solo llega a un 7\%, mientras que el comñun de las ciencias sociales apenas sobrepasa un $10 \%$.

\section{Referencias}

Alhuay Quispe, J., \& Pacheco Mendoza, J. (2018). Escaso uso de indicadores de productividad científica en estudios bibliométricos. Educación Médica .

Alonso García, S., \& Alonso García, M. d. (2014). Las Redes Sociales en las Universidades Españolas. Revista de Comunicación Vivat Academia (126), 54-62.

Cetto, A. (2012). Los indicadores bibliométricos y los rankings de universidades. En U. A. Domingo (Ed.), Seminario " "Las universidades dominicanas frente a los rankings internacionales". Santo Domingo: Centro Latindex para República Dominicana; Universidad APEC. 
Chinchilla-Rodríguez, Z. (2018). Indicadores bibliométricos en Humanidades y Ciencias Sociales: una perspectiva comparada. Scientometrics .

Clarivate Analytics. (s.f.). Web Of Science. Recuperado el 1 de 8 de 2018, de https://apps.webofknowledge.com/

Dorta-Contreras, A. J. (2018). Ciencia a la medida. Estudios bibliométricos y cienciométricos en una nueva sección Science with measures. (U. d. (LABCEL), Ed.) ecimed Revista Habanera de Ciencias Médicas , 17 (4), 508-509.

Galindo Rodríguez, A., \& Arbinaga, F. (2018). Análisis bibliométrico de la revista iberoamericana de psicología del ejercicio y el deporte (2006-2017). Revista Iberoamericana de Psicología del Ejercicio y el Deporte, 13 (1), 33-40.

Garrigos-Simón, F. J., Oltra Gutiérrez, J. V., \& Narangajavana, Y. (2016). Ventajas y usos de Twitter, como herramienta de mejora de la educación universitaria. IN RED (págs. 1-10). Valecia: Universitat Politécnica de Valéncia.

Guzmán Duque, A. P., Del Moral Pérez, M. E., \& González Ladrón de Guevara, F. (2012). Usos de Twitter en las universidades iberoamericanas . RELATEC , 27-41.

Kassens-Noor, E. (2012). Twitter as a Teaching Practice to Enhance Active and Informal Learning in Higher Education: The Case of Sustainable Tweets. Active Learning in Higher Education , 13 (1), 9-21.

Langheinrich, M. (2018). Bibliometrics-Measuring Pervasive Computing Impact. IEEE Pervasive Computing , 6-9.

Luo, F., Sun, A., Erdt, M., Raamkumar, A. S., \& Theng, Y.-L. (2018). Exploring prestigious citations sourced from top universities in bibliometrics and altmetrics: a case study in the computer science discipline. Scientometrics .

Veletsianos, G. (2012). Higher education scholars' participation and practices on Twitter. Journal of Computer Assisted Learning , 28, 336-349. 